\title{
Erratum to: Heart rate variability in male shift workers in automobile manufacturing factories in South Korea
}

\author{
Sangyoon Lee · Ho Kim • Dae-Hwan Kim • \\ Myunggul Yum $\cdot$ Mia Son
}

Published online: 27 February 2015

(C) Springer-Verlag Berlin Heidelberg 2015

\section{Erratum to: Int Arch Occup Environ Health DOI 10.1007/s00420-014-1016-8}

Unfortunately, the author affiliations were omitted in the original publication. The complete list of author affiliations should read as below:

\section{Sangyoon Lee, Dae-Hwan Kim}

Department of Occupational and Environmental Medicine, Haeundae Paik Hospital, Inje University, 875, Haeun-daero, Haundae-gu, Busan, 612-896, Republic of Korea

The online version of the original article can be found under doi:10.1007/s00420-014-1016-8.

S. Lee $\cdot$ D.-H. Kim

Department of Occupational and Environmental Medicine, Haeundae Paik Hospital, Inje University, 875, Haeun-daero, Haundae-gu, Busan 612-896, Republic of Korea

\section{H. Kim}

Department of Biostatistics and Epidemiology, School of Public

Health, Seoul National University, 1 Gwanak-ro, Gwanak-gu,

Seoul 151-742, Republic of Korea

\section{Yum}

Department of Pediatrics, College of Medicine, Hanyang University, Kyoungchun-ro 153, Guri-si, Gyeonggi-do 471-701, Republic of Korea

\section{Son ( $\square)$}

Department of Preventive Medicine, School of Medicine, Kangwon National University, 1 Kangwondaehak-gil, Chuncheon-si, Gangwon-do 200-701, Republic of Korea e-mail: sonmia@kangwon.ac.kr
Ho Kim

Department of Biostatistics and epidemiology, School of Public Health, Seoul National University, 1 Gwanak-ro, Gwanak-gu, Seoul, 151-742, Republic of Korea

Myunggul Yum

Department of Pediatrics, College of Medicine, Hanyang University, Kyoungchun-ro 153, Guri-si, Gyeonggi-do, 471-701, Republic of Korea

\section{Mia Son}

Department of Preventive Medicine, School of Medicine, Kangwon National University, 1 Kangwondaehak-gil, Chuncheon-si, Gangwon-do, 200-701, Republic of Korea 